\title{
A saúde na perspectiva da comunicação institucional e mercadológica
}

Health under the perspective of institutional and marketing communication

La salud en la perspectiva de la comunicación institucional y mercadológica

\section{Angela Lovato Dellazzana}

- Doutoranda em Comunicação Social na Pontifícia Universidade Católica do Rio Grande do Sul (PUC-RS)

- Fez estágio de doutorado em Comunicação Social na Universidad de Navarra (Espanha)

- Especialista em Marketing pela PUC-RS

- Professora do Centro Universitário Franciscano (Unifra), de Santa Maria (RS)

- Foi assessora de Comunicação e Marketing do Hospital Ernesto Dornelles, de Porto Alegre (RS)

- E-mail: angela.lovato@terra.com.br 


\section{Resumo}

O presente estudo baseia-se em um levantamento bibliográfico das pesquisas publicadas em 2011 com o tema comunicação e saúde, nos portais da Sociedade Brasileira de Estudos Interdisciplinares da Comunicação (Intercom) e da Associação Nacional dos Programas de Pós-Graduação em Comunicação (Compós). Apresenta-se uma pesquisa exploratória, de caráter quantitativo e qualitativo, da diversificada bibliografia encontrada sobre o tema, relacionando-a com o conceito de comunicação organizacional integrada, desenvolvido por Margarida Kunsch. O primeiro passo foi identificar as possíveis relações que podem ser estabelecidas entre os termos comunicação e saúde. Percebe-se que o tema engendra vieses os mais diversos e que, na maioria dos casos, a comunicação organizacional em saúde privilegia o aspecto institucional em detrimento do mercadológico.

PALAVRAS-CHAVE: COMUNICAÇÃO INTEGRADA • COMUNICAÇÃO INSTITUCIONAL • COMUNICAÇÃO MERCADOLÓGICA•ORGANIZAÇÕES DE SAÚDE

\section{Abstract}

This study is based on a bibliographical assessment of the surveys published in 2011 on the theme of communication and health, in the portals of the Brazilian Society of Interdisciplinary Studies of Communication (Intercom) and of the National Association of Communication Postgraduate Programs (Compós). An exploratory survey of a quantitative and qualitative nature is presented of the diversified bibliography found on the matter, relating it to the concept of integrated organizational communication developed by Margarida Kunsch. The first step was to identify the possible relations that could be established between the terms communication and health. It is perceived that engender biases of various kinds and that, in most cases, the organizational communication on health privileges the institutional aspect in detriment of marketing.

KEYWORDS: INTEGRATED COMMUNICATION • INSTITUTIONAL COMMUNICATION • MARKETING COMMUNICATION • HEALTH ORGANIZATIONS

\section{Resumen}

Este estudio se fundamenta en un levantamiento bibliográfico de las investigaciones publicadas en 2011 con el tema comunicación y salud, en los portales de los sitios de la Sociedad Brasilera de Estudios Interdisciplinarios de la Comunicación (Intercom) y de la Asociación Nacional de los Programas de Pos-Grado en Comunicación (Compós). Se presenta una investigación exploratoria, de carácter cuantitativo y cualitativo, de la diversa bibliografía encontrada sobre el tema, relacionándola con el concepto de comunicación organizacional integrada, desarrollado por Margarida Kunsch. El primer paso fue identificar las posibles relaciones que pueden ser establecidas entre los términos comunicación y salud. Se constata que el tema engendra los más diversos desdoblamientos y que, en la mayoría de los casos, la comunicación organizacional en salud privilegia el aspecto institucional en detrimento de lo mercadológico.

PALAVRAS CLAVE: COMUNICACIÓN INTEGRADA • COMUNICACIÓN INSTITUCIONAL • COMUNICACIÓN MERCADOLÓGICA • ORGANIZACIONES DE SALUD 
A área da saúde representa um desafio para os profissionais da comunicação organizacional. Diferentemente de outras áreas, como a indústria e o comércio de bens de consumo duráveis, por exemplo, as empresas relacionadas à área da saúde, aqui entendida como clínicas, laboratórios e hospitais, apresentam restrições no que tange à comunicação com seus diversos públicos de interesse. A principal ressalva se dá em função da natureza desses serviços, que têm sua divulgação regulada por órgãos profissionais, como o Conselho Federal de Medicina, que estabelece: "A publicidade médica deve obedecer exclusivamente a princípios éticos de orientação educativa, não sendo comparável à publicidade de produtos e práticas meramente comerciais" (CFM, 2012, p. 1).

O Conselho Regional de Medicina do Rio Grande do Sul (Cremers) possui uma comissão especial que tem por finalidade "supervisionar qualquer divulgação médica, através dos meios de comunicação, feita por estabelecimento de saúde, órgão governamental ou privado" (Cremers, 2012, s. p). Dessa forma, os profissionais que trabalham na comunicação dessas instituições devem estar atentos a essas restrições, pois nem todas as práticas de comunicação organizacional empregadas tradicionalmente podem ser aplicadas à área da saúde. É importante salientar que a comunicação organizacional, neste estudo, é entendida a partir da perspectiva de Margarida Kunsch (2007) ${ }^{1}$, que considera seus diversos âmbitos: a comunicação interna e administrativa, a comunicação institucional e a comunicação mercadológica.

A experiência de oito anos na assessoria de comunicação e marketing de um grande hospital há muito despertou em nós o interesse em pesquisar academicamente a relação entre a comunicação e a saúde. Por diversas razões de ordem prática, o desejo permaneceu latente e agora recebe um estímulo para ser concretizado, ainda que em caráter embrionário, graças à oportunidade lançada pela revista Organicom, que no dossiê desta 16a. edição apresenta a temática comunicação e saúde.

Buscou-se realizar um levantamento bibliográfico que permitisse apreender o estado da arte nessa área, como primeira etapa de uma pesquisa que se pretende desenvolver ao longo do ano 2012. Trata-se, aqui, de apresentar um estudo exploratório, de caráter quantitativo e qualitativo, da diversificada bibliografia encontrada sobre o tema. O primeiro passo foi identificar as possíveis relações que podem ser estabelecidas entre os termos comunicação e saúde. E já de início encontrou-se uma afirmação que pôde ser confirmada ao longo das leituras realizadas: "No que diz respeito às relações estabelecidas entre comunicação e saúde, poderíamos apontar que se trata de algo mais complexo e mais antigo do que, à primeira vista, podemos supor" (Trench; Antenor, 2010, p. 4).

\footnotetext{
1 Trata-se de capítulo de Kunsch em livro organizado por Clotilde Pérez e Ivan S. Barbosa (2007). A autora apresentou sua visão pioneira da "comunicação organizacional integrada" em livro de 1986 (Kunsch, 1986), que teve uma 4a. edição, revista, atualizada e ampliada em 2003 (Kunsch, 2003). Nesta última edição, ela explora a temática no capítulo "Relações públicas e a filosofia da comunicação integrada" (Kunsch, 2003, p. 149-202). A obra está na 5a. edição.
} 
Na área da saúde, destaque-se José C. Teixeira (2004, p. 615), que utiliza o termo comunicação em saúde com a seguinte definição: "Estudo e utilização de estratégias de comunicação para informar e para influenciar as decisões dos indivíduos e das comunidades no sentido de promoverem a sua saúde". O autor aborda o tema no âmbito da psicologia e considera a comunicação como um aspecto transversal em saúde, com relevância em diversos contextos. Contudo, a comunicação organizacional não é contemplada em sua plenitude, uma vez que Teixeira apenas considera a comunicação interna e administrativa das organizações de saúde, excluindo a comunicação institucional e mercadológica, ou seja, as práticas que visam trabalhar a imagem e a publicidade dos serviços dessas instituições perante seus públicos.

Essa perspectiva da relação entre comunicação e saúde parece ser a mais recorrente na bibliografia encontrada. Graciela Natansohn (2004, p. 39), que desenvolve o tema a partir da área da comunicação, também aborda a questão enfocando a perspectiva das políticas públicas de saúde. Para essa autora, o tema passa a ser importante na América Latina a partir da década 1960, época em que

a comunicação e a saúde, junto à educação, passam a ter um vínculo estreito: tratava-se de superar a 'ignorância' do povo, a resistência às inovações - especialmente, da população rural - mediante a educação e o planejamento descentralizado e regionalizado.

A autora aponta a tradução para o espanhol do livro Mass media and national development, de Wilbur Schramm, feita em 1964, como um marco do enfoque desenvolvimentista e instrumental de que a comunicação desfrutava no continente.

Assim, entende-se que o tema é vasto e engendra vieses os mais diversos, tendo caráter ora sanitarista, ora marxista, ora mercadológico, para citar alguns exemplos. No entanto, não se pretende, ao menos nesta etapa, desenvolver, sustentar ou criticar os pontos de vista encontrados, mas relatá-los e relacioná-los à perspectiva da comunicação organizacional integrada de Kunsch (2007). Obviamente, o estudo dessas relações é preocupação tanto da área da saúde quanto da área da comunicação. Contudo, com vistas a manter o foco deste trabalho sob o olhar da comunicação, o corpus de pesquisa restringiu-se às publicações ${ }^{2}$ do ano 2011 da Sociedade Brasileira de Estudos Interdisciplinares da Comunicação (Intercom) e da Associação Nacional dos Programas de Pós-Graduação em Comunicação (Compós), uma vez que estas têm forte expressão nacional. $\mathrm{Na}$ etapa quantitativa do levantamento bibliográfico, foram consultados os sítios dessas instituições na internet e foi digitado nos respectivos buscadores a palavra "saúde"3. Em seguida, foi feita a etapa qualitativa, que consiste em uma leitura desses trabalhos para relacioná-los ao conceito da comunicação organizacional integrada.

\footnotetext{
2 Concretamente, às publicações disponibilizadas virtualmente, com acesso aberto nos portais das organizações.

3 No sítio da Intercom, disponível em <http://www.portalintercom.org.br>, as buscas foram feitas nos menus "Anais" e " Publicações". No sítio da Compós
} 


\section{PONTO DE PARTIDA: A COMUNICAÇÃO ORGANIZACIONAL INTEGRADA}

Kunsch (2007, p. 371) critica o caráter meramente instrumental da comunicação organizacional, dominante ainda na metade final do século XX no Brasil. Para a autora: "há a necessidade (...) de trabalharmos a comunicação nas organizações numa perspectiva muito mais interpretativa do que instrumental e com base em uma visão de mundo". O conceito de comunicação organizacional integrada que ela desenvolveu não exclui seu caráter instrumental, mas o relaciona com a complexidade da natureza das organizações e da própria comunicação interpessoal.

A integração se dá a partir do pressuposto de que os quatro âmbitos da comunicação organizacional identificados inicialmente pela autora se inter-relacionam de forma a destacar duas áreas fundamentais: as relações públicas e o marketing. "A primeira abarcaria, pela sua essência teórica, a comunicação institucional, a comunicação interna e a comunicação administrativa. $O$ marketing responderia por toda a comunicação mercadológica" (Kunsch, 2007, p. 386). A autora esclarece que o intuito dessa integração não se restringe ao sucesso da organização isoladamente, seja qual for seu ramo e sua natureza, mas visa também ao benefício de seus diversos públicos e da sociedade em geral.

Nesse sentido, Cicilia Krohling Peruzzo (1993), já no começo da década de 1990, abordava a temática da saúde na perspectiva da comunicação comunitária, como um instrumento a serviço do desenvolvimento e em prol da sociedade. A autora combina os temas relações públicas, movimentos populares e transformação social, afirmando que a comunicação, se bem empregada, pode contribuir para a formação da cidadania, organizando a população para que reivindique seu acesso aos serviços básicos de saúde, por exemplo. Portanto, o enfoque do tema saúde desenvolvido pela autora também é claramente voltado para a saúde pública e para as políticas de comunicação, enfatizando o âmbito da comunicação institucional.

Arquimedes Pessoni (2008, p. 177) emprega o termo "comunicação para a saúde" nessa mesma perspectiva, fazendo um resgate da pesquisa acadêmica sobre o tema. Para esse autor, "não há concordância sobre a data em que se iniciam os estudos da comunicação para a saúde como disciplina. Há, isso sim, alguns fatos que contribuíram para que a comunicação fosse vista com mais atenção pelos profissionais de saúde e vice-versa". É possível perceber que Pessoni considera a relação entre a comunicação e a saúde tanto a partir da saúde quanto a partir da comunicação. Destacam-se, em sua abordagem, os relatos sobre a criação e as edições da Conferência Brasileira de Comunicação e Saúde (Comsaúde), que reúne profissionais de ambas as áreas.

Lia Luz (2010), por sua vez, aborda a relação entre comunicação e saúde a partir do jornalismo científico, categorizando cinquenta matérias da revista Veja que traziam o tema saúde. A autora 
mostra que a maior parte das reportagens se concentrava em questões alimentares, com 15\% de frequência: "Em seis meses, Veja apresentou sete matérias sobre regimes e formas de perder peso" (Luz, 2010, p. 265). No entanto, Lia deixa claro que a revista não visa apenas conscientizar a população sobre os perigos da obesidade, uma vez que cita medicamentos e laboratórios farmacêuticos nessas reportagens.

Nesse sentido, identifica-se outro ponto de vista sobre a relação entre comunicação e saúde, o do jornalismo científico, atividade que pode implicar interesses de lucro implícitos, conforme conclusão de Luz (2010, p. 273): "Pode-se afirmar que a saúde se tornou, em muitos casos, uma mercadoria que pode ser comprada na farmácia da esquina”. Nesse caso, percebe-se que a comunicação institucional acaba por mesclar-se com a comunicação mercadológica, ainda que de forma velada.

Sobre o jornalismo científico é interessante destacar a visão de José A. de Oliveira e Isaac Epstein (2009), que afirmam que a ciências tornou atraente para a opinião pública, grande parte em função do jornalismo científico e da divulgação científica. Ressaltem-se os objetivos de seu estudo, que visa analisar

a assimétrica relação entre o tempo da pesquisa científica e o tempo dos diferentes segmentos interessados em seus resultados, sobretudo com a crescente demanda de se construir consenso técnico sobre campos da ciência que exigem rigorosas investigações e exaustivos testes. Destacam-se, sobretudo nos últimos anos, as pressões de setores da sociedade civil, interessados em participar do processo decisório que orienta os rumos da ciência, entre eles: o jornalismo científico, o poder legislativo e a opinião pública (Oliveira; Epstein, 2009, p. 423).

Os autores explicam que essa assimetria se deve ao seguinte impasse: o público busca no trabalho da ciência "verdades" permanentes, soluções definitivas. A ciência, por sua vez, aponta para certezas provisórias que poderão ser confirmadas, refutadas ou transformadas conforme o saber científico for sendo construído. Concorrem para a problematização da questão os diversos interesses financeiros na divulgação de determinadas pesquisas científicas. Assim, surge a polêmica relacionada ao abuso cometido pelo jornalismo científico, que muitas vezes é acusado de escrever matérias permeadas de intenções ocultas de venda, como relata Luz (2010).

Entende-se que esse uso do jornalismo científico com intenções de venda estaria relacionado à própria transformação da saúde em "mercadoria", como mencionada pela autora, constatação que encontra respaldo em Murilo C. Ramos (2005, p.250): 
Como bem expresso em documento fundador do Laboratório de Políticas Públicas (LPP), da Universidade Estadual do Rio de Janeiro (ver http://www2.uerj.br/lpp), na medida em que o mercado não reconhece direitos, a função central que ele vai assumindo na reformulação das relações econômicas e sociais representa uma transformação do que era direito em um bem negociável no mercado. Assim, de direitos universais os direitos à educação e à saúde passaram a ser mercadorias, e, concomitantemente, o Estado deixou de desempenhar seu papel na afirmação de direitos, para, ao contrário, centrando-se em políticas de desregulamentação, abrir espaços para a mercantilização crescente das políticas sociais.

Assim, percebe-se que as pesquisas envolvendo a relação entre a comunicação e a saúde engendram vieses que enfocam ora a comunicação institucional, ora a comunicação mercadológica, dependendo do ponto de partida do pesquisador. Cada um desses vieses pode gerar estudos diversos, tamanha a abrangência do tema. Buscou-se demonstrar, até este ponto, que se torna evidente a necessidade de realizar um compêndio sobre o estado da arte desses estudos sob a ótica da comunicação integrada. Dessa forma, identificam-se a seguir os âmbitos da comunicação organizacional integrada presentes nas publicações da área da comunicação sobre o tema saúde, em 2011, nos portais da Intercom e da Compós.

\section{O ESTADO DA ARTE}

Não foram encontradas publicações datadas de 2011 com o tema saúde no sítio da Compós. As buscas por esse indexador foram feitas no menu "Biblioteca" (há apenas três publicações com o tema saúde - duas em 2001 e uma em 2002) e no menu E-compós (há apenas duas publicações 2004 e 2010). No livro da Compós 2011 (Silva et al., 2011), disponível para download, também não foi encontrada essa temática. Acredita-se que a pouca preocupação com o tema na Compós pode estar relacionada com o intuito da associação de dar prioridade aos temas mais relevantes para a área, sendo que a saúde não parece estar entre eles. De fato, a saúde não possui um grupo de trabalho (GT) próprio na associação e nem aparece diretamente como assunto na descrição de algum dos quinze grupos ativos. As publicações encontradas no menu "Biblioteca" sobre o tema saúde, nos anos 2001 e 2002, foram apresentadas no GT de Estratégias e Políticas de Comunicação, que, talvez por sua abrangência excessiva, foi desmembrado e encontra-se inativo.

Nas consultas realizadas no sítio da Intercom, foram encontrados 29 estudos com o tema saúde publicados em 2011, conforme o quadro 1. 
Quadro 1 - Publicações sobre comunicação e saúde em 2011

\begin{tabular}{|c|}
\hline MENU ACESSADO / TÍTULO DO TEXTO \\
\hline Revista Iniciacom \\
\hline $\begin{array}{l}\text { 1. Saúde em revista: análise dos temas das reportagens das publicações empresariais Essência } \\
\text { e Unimed }\end{array}$ \\
\hline Anais do Intercom Sudeste \\
\hline 2. Um novo eu: cirurgia plástica e as histórias que transformam vidas \\
\hline Anais do Intercom Sul \\
\hline $\begin{array}{l}\text { 3. Assessoria de imprensa para a academia Vidativa } \\
\text { 4. Site Prosa \& Prozac: produção jornalística sobre saúde } \\
\text { 5. Propaganda de medicamentos: faz mal à saúde? } \\
\text { 6. Olhares sobre a divulgação científica de saúde: uma análise do jornalismo realizado pela } \\
\text { Superinteressante e Galileu de } 2009 \text { a } 2010 \\
\text { 7. Publicidade e Aids: análise dos comerciais veiculados pela mídia eletrônica } \\
\text { 8. "Passaporte para a cidadania": a comunicação comunitária na construção de uma política } \\
\text { sindical na área da saúde e segurança no trabalho }\end{array}$ \\
\hline Anais do Intercom Norte \\
\hline 9. Radiojornal Saúde \& Você \\
\hline Anais do Intercom Centro-Oeste \\
\hline $\begin{array}{l}\text { 10. Coisa doida: comunicação e saúde mental } \\
\text { 11. Uma experiência em assessoria de comunicação para a Maternidade Nossa Senhora de } \\
\text { Lourdes em Goiânia } \\
\text { 12. Viva melhor: qualidade de vida e nutrição } \\
\text { 13. Comunicação social e educação popular de jovens: um desafio para o SUS }\end{array}$ \\
\hline Anais do Intercom Nordeste \\
\hline $\begin{array}{l}\text { 14. Uma leitura da apologia do estatuto da magreza na revista Veja } \\
\text { 15. Plano de Comunicação para o Hospital Infantil Varela Santiago }\end{array}$ \\
\hline Anais do Intercom Nacional \\
\hline $\begin{array}{l}\text { 16. A invisibilidade negra: o (des)encontro do jornalismo com saúde pública nas doenças de } \\
\text { recorte étnico-racial e por vulnerabilidade que acometem a população afrodescendente } \\
\text { 17. A temática "saúde" na pauta da Intercom: análise dos anais dos congressos de } 2000 \text { a } 2010 \\
\text { 18. A verificação da produção científica na área da comunicação e saúde em quatro pe- } \\
\text { riódicos científicos }\end{array}$ \\
\hline
\end{tabular}


19. Dilemas sociais em campanhas de saúde pública: comparação entre conteúdos de campanhas públicas recentes

20. Entre invisibilidades e movimentos: as pessoas com deficiência na reivindicação do acesso à informação, cidadania e direito à saúde

21. Observatório Saúde na mídia: um olhar sobre o gênero editorial dos jornais

Folha de Pernambuco e Jornal do Commercio

22. Saúde masculina: invisível até na tv

23. Tabagismo em diálogo: olhares sobre o discurso do Ministério da Saúde.

24. A cobertura da mídia impressa da dengue em Mato Grosso do Sul

25. A "salvação" nos remédios: análise do fenômeno da medicalização no discurso midiático

26. Análise das relações comunicacionais em uma organização hospitalar à luz da autopoiese

27. O uso das mídias sociais pela Secretaria de Estado de Saúde de Minas Gerais (SES-MG)

28. Os usos do cuidado de si na produção de informação

29. Percepção das campanhas de prevenção às doenças sexualmente transmissíveis (DST): o caso da comunidade do Orkut "Papiloma vírus humano"

Ao fazer uma breve leitura dos 29 textos publicados sobre o tema comunicação e saúde, foi possível identificar sete diferentes abordagens, por ordem de aparecimento: 1) comunicação das empresas da saúde com seus públicos; 2) jornalismo sobre beleza/estética; 3)jornalismo científico sobre doenças/prevenção; 4) campanhas de saúde pública/conscientização; 5) políticas de comunicação/comunicação comunitária; 6) propaganda de medicamentos; 7) pesquisas sobre a comunicação e a saúde.

Como já se esperava, o enfoque mais frequente esteve na questão da saúde pública, que utiliza a comunicação em campanhas de conscientização da população quanto à prevenção e tratamentos de doenças em geral; ele apareceu em oito casos (estudos 7, 19, 22, 23, 24, 27, 28 e 29). Houve um empate no segundo enfoque mais presente, que aparece seis vezes: um dizia respeito às políticas de comunicação, à comunicação comunitária e ao acesso à informação de serviços de saúde (estudos $8,10,12,13,16$ e 20); e outro, à comunicação das empresas da aérea da saúde com seus públicos de interesse (estudos 1, 3, 9, 11, 15, 26). Em terceiro lugar esteve a questão do jornalismo científico na área da saúde, com matérias sobre novos medicamentos e tratamentos, por exemplo (estudos 4, 6, 21). Em quarto lugar, ficaram empatados os estudos sobre a saúde e a beleza (estudos 2 e 14), sobre propaganda e divulgação de medicamentos (estudos 5 e 25) e sobre as pesquisas em comunicação e saúde (estudos 17 e 18).

Entre os sete enfoques identificados, aquele que mais interessa a este estudo, pois está mais relacionado com o conceito de comunicação organizacional integrada, é o do relacionamento das organizações que prestam serviços de saúde com seus públicos de interesse. Os seis estudos com esse enfoque são descritos a seguir. 
No estudo 1, "Saúde em revista: análise dos temas das reportagens das publicações empresariais Essência e Unimed", Felipe da Costa e Valquíria John (2011), fazem uma análise de conteúdo de jornais empresariais de prestadoras de serviços de saúde, respectivamente, o Hospital Santa Catarina o plano de saúde Unimed Blumenau. A intenção dos autores foi categorizar os temas mais recorrentes para identificar como se dá o relacionamento dessas empresas com seus clientes por meio desses veículos. Para os autores,

a união dessas duas especialidades do jornalismo, empresarial e de saúde, se observadas suas características essenciais, pode trazer benefícios para o emissor, que reforça sua marca, e para o receptor, que se informa sobre hábitos que podem melhorar sua saúde (Costa; John, 2011, p. 2).

Apesar de não haver menção ao conceito de comunicação integrada, percebe-se que, no referido estudo, a comunicação organizacional é enfocada sob o viés da comunicação institucional. A comunicação é vista pelos autores como benéfica tanto para a empresa quanto para seus clientes, por meio de veículos empresariais que visam reforçar a marca e o relacionamento com seus usuários. Essa afirmação é comprovada na análise dos autores, que concluem que a prevenção de doenças é um dos temas mais recorrentes nas duas publicações, demonstrando a preocupação das empresas em levar informação de educação em saúde para seus leitores.

O estudo 3, "Assessoria de imprensa para a academia Vidativa", de Ana Paula L. Kringel e Taiane de Oliveira Volcan (2011), traz uma proposta de implantação de assessoria de imprensa para uma academia na cidade de Pelotas (RS), enfocando a necessidade de criar um veículo interno para a empresa. Nesse sentido, o âmbito da comunicação organizacional priorizado pelos autores é a comunicação interna. Contudo, o trabalho tem o intuito de apresentar apenas uma proposta e, sendo assim, não traz informações sobre a aplicação e os resultados desse caso, nem relaciona teoricamente os conceitos de comunicação integrada com os objetivos do trabalho.

Já o estudo 9, "Radiojornal Saúde \& Você", de autoria de Cynthia Pinheiro et al. (2011, p. 3) traz uma perspectiva da comunicação organizacional voltada tanto para a comunicação institucional quanto para a comunicação interna, uma vez que retrata os objetivos desse veículo: "Suprir a necessidade de melhorar a comunicação entre os usuários e funcionários do HUGV [e] contribuir para difundir junto aos pacientes e seus familiares informações sobre como cuidar da saúde e ter uma melhor qualidade de vida". Contudo, o artigo apenas cita a comunicação integrada quando relata uma pesquisa feita por outro hospital.

O estudo 11,"Uma experiência em assessoria de comunicação para a Maternidade Nossa Senhora de Lourdes em Goiânia”, de Ana Carolina R. de Deus et al. (2011), é um relato de um projeto experimental que, já na introdução, demonstra preocupação com o conceito de comunicação organizacional integrada: 
Em tempos de globalização, de discussão acerca da consolidação dos direitos sociais e da cidadania, o conceito de comunicação organizacional não cabe apenas às empresas da iniciativa privada, mas aplica-se a todo tipo de organização, inclusive às organizações públicas (Deus et al., 2011, p. 1).

No entanto, os autores não desenvolvem os conceitos de comunicação integrada ao longo do trabalho.

O estudo 15, "Plano de comunicação para o Hospital Infantil Varela Santiago", de José Alves de Souza e Maria Stella Galvão Santos, também traz o conceito de comunicação integrada, mas não demonstra sua aplicabilidade no objeto estudado.

Por fim, o estudo 26, "Análise das relações comunicacionais em uma organização hospitalar à luz da autopoiese", de Michele Paris, visa compreender os fatores que influenciam a comunicação organizacional na Santa Casa de Misericórdia de Maceió (AL). A autora entende que

A comunicação organizacional, principalmente na saúde, surge não só como uma estratégia para prover indivíduos e coletividade de informações, pois se reconhece que a informação não é suficiente para favorecer mudanças, mas é uma chave, dentro do processo educativo, para compartilhar conhecimentos e práticas que podem contribuir para a conquista de melhores condições de vida (Paris, 2011, p. 4).

Sob essa ótica, Paris privilegia o âmbito da comunicação interna e da comunicação institucional, não referenciando o conceito de comunicação integrada de Kunsch (2007).

A partir dessa breve análise dos estudos de comunicação que permeiam o tema da saúde, é possível perceber o pouco interesse existente entre os pesquisadores de comunicação pelos conceitos de comunicação integrada aplicados à área da saúde. Percebe-se, ainda, que nenhum estudo enfocou o âmbito da comunicação mercadológica, aspecto que, muitas vezes, é desestimulado pelas entidades de classe da área da saúde e carece de pesquisas.

\section{CONSIDERAÇÕES FINAIS}

O desafio proposto por este estudo permitiu trilhar um primeiro passo em busca da compreensão sobre a tensa relação entre os termos comunicação e saúde. Identificaram-se seis abordagens possíveis para nortear a pesquisa sobre o tema, constatando-se a complexidade e a diversidade de enfoques que esse debate pode suscitar. Nessas diferentes abordagens, buscou-se encontrar o viés da comunicação organizacional integrada, que não foi explorado de maneira satisfatória nos estudos analisados. De certa forma, por se tratar de pesquisas, em 
sua maioria, de iniciação científica, careceram de um amparo teórico consistente. Essa constatação revela o descaso sobre o tema de pesquisadores já experientes, mas, por outro lado, revela que são jovens estudantes que se interessam pelo tema, o que pode representar um futuro de pesquisas mais assíduas nessa área.

A prevalência dos âmbitos da comunicação institucional e interna já era esperada como resultado desta pesquisa, uma vez que a aproximação dos conceitos de comunicação mercadológica e serviços de saúde é recente e ainda suscita desconfianças por parte do público e dos próprios gestores desses serviços. Contudo, a necessidade de gestão da comunicação de instituições de saúde, por exemplo, já é ponto pacífico, pois muitas instituições hospitalares, sejam públicas ou privadas, já dispõem de assessorias ou departamentos de comunicação. Isso também é percebido na maioria dos estudos encontrados, que tratam de relatos de casos de assessorias de comunicação de hospitais, visando ora à melhora da qualidade do trabalho (comunicação interna), ora à criação e manutenção de uma imagem positiva junto a seus usuários (comunicação institucional).

Todavia, abordou-se apenas um enfoque do diverso leque de possibilidades que o tema engendra e que ainda carecem de pesquisas. Acredita-se que o estudo das relações entre comunicação e saúde se faz necessário tanto na área da comunicação organizacional quanto na área da comunicação comunitária e da comunicação para a cidadania, perpassando enfoques como a construção de políticas públicas de regulamentação da publicidade de serviços de saúde. Este enfoque, a publicidade de entidades prestadoras de serviços de saúde, por exemplo, não apareceu nos estudos analisados, ainda que o tema seja atual, haja vista as crescentes regulamentações sobre essa atividade.

Tal constatação evidencia a necessidade de debater o tema de forma sistemática. O fato é que, devido à complexidade das questões ligadas à comunicação organizacional de empresas prestadoras de serviços de saúde, é premente uma abordagem teórica mais consistente. Além disso, se faz mister uma análise empírica que permita mapear e sugerir caminhos para a complexa realidade que os profissionais de comunicação enfrentam quando o objeto de sua prática é a saúde.

\section{REFERÊNCIAS}

CFM - Conselho Federal de Medicina. Resolução CFM n 1.701/2003. Disponível em: <http://www.portalmedico.org.br/ resolucoes/CFM/2003/1701_2003.htm>. Acesos em: 10 jan. 2012.

COSTA, Felipe da; JOHN, Valquíria Michela. Saúde em revista: análise dos temas das reportagens das publicações empresariais Essência e Unimed. Iniciacom - Revista Brasileira de Iniciação Científica em Comunicação Social, São Paulo, Intercom, v. 3, n. 1, abr. 2011. Disponível em: <http:// www.portcom.intercom.org.br/ojs-2.3.1-2/index.php/iniciacom/article/ view/620/580>. Acesso em: 01 mar. 2012.

CREMERS - Conselho Regional de Medicina do Rio Grande do Sul. Comissão de Divulgação de Assuntos Médicos (Codame). Propaganda em medicina. Disponível em: <http://www.cremers.org.br/index.php? indice=74>. Acesso em: 25 jan. 2012. 
DEUS, Ana C. Rodrigues de et al. Uma experiência em assessoria de comunicação para a Maternidade Nossa Senhora de Lourdes em Goiânia. In: CONGRESSO DE COMUNICAÇÃO DA REGIÃO CENTRO-OESTE, XIII, Cuiabá (MT), 08 a 10 jun. 2011. Anais... São Paulo: Intercom, 2011. Disponível em: <http://intercom.org.br/papers/regionais/centrooeste2011/ expocom/EX27-0205-1.pdf>. Acesso em: 10 jan. 2012.

KRINGEL, Ana Paula L.; VOLCAN, Taiane de Oliveira. Assessoria de imprensa para a academia Vidativa. In: CONGRESSO DE COMUNICAÇÃO DA REGIÃO SUL, XII, Londrina (PR), 26 a 28.05.2011. Anais... São Paulo: Intercom, 2011.

KUNSCH, Margarida M. Krohling. Comunicação organizacional: surgimento e evolução das práticas, conceitos e dimensões. In: PEREZ, Clotilde; BARBOSA, Ivan Santo (Org.). Hiperpublicidade. Vol. 1. Fundamentos e interfaces. São Paulo: Thomson Learning, 2007.

Planejamento de relações públicas na comunicação integrada. [1986]. 4. ed. - rev., atual. e ampl. São Paulo: Summus, 2003.

LUZ, Lia. A pílula da longevidade à venda nas páginas da revista Veja. In: Revista Brasileira de Ciências da Comunicação, São Paulo, Intercom, v. 33 n. 1, 2010. Disponível em: <http://www.portcom.intercom.org.br/ojs-2.3.1-2/index.php/revistaintercom/article/view/156/149>. Acesso em: 25 jan. 2012.

NATANSOHN, Graciela. Comunicacão \& Saúde: interfaces e diálogos possíveis. Revista de Economia Política de las Tecnologías de la Información y Comunicación, Eptic, v. VI, n. 2, mayo-ago. 2004. Disponível em: <http://www2.eptic.com.br/ sgw/data/bib/artigos/ac91b84bc163228f74ae2a291b80dd81.pdf>. Acesso em: 04 fev. 2012.

OLIVEIRA, José Aparecido de; EPSTEIN, Isaac. Tempo, ciência e consenso: os diferentes tempos que envolvem a pesquisa científica, a decisão política e a opinião pública. Interface - Comunicação, Saúde, Educação, v. 13, n. 29, p. 423-33, abr.-jun. 2009. Disponível em: <http://www.scielo.br/pdf/icse/ v13n29/v13n29a14.pdf>. Acesso em: 20 mar. 2011.

PARIS, Michele. Análise das relações comunicacionais em uma organização hospitalar à luz da autopoiese. In: CONGRESSO BRASILEIRO DE CIÊNCIAS DA COMUNICAÇÃO, XXXIV, Recife (PE), 02 a 06 set. 2011. Anais... São Paulo: Intercom, 2011. Disponível em http://www.intercom.org.br/papers/ nacionais/2011/resumos/R6-1309-1.pdf Acesso 12 jan. 2012.

PERUZZO, Cicilia M. Krohling. Revista Brasileira de Comunicação, São Paulo, Intercom, v. XVI, n. 2, p. 125-133, 1993. Disponível em: <http://www.bocc.ubi.pt/pag/peruzzo-cicilia-relacoes-publicas.pdf>. Acesso em: 30 jan. 2012.

PESSONI, Arquimedes. Comunicação para a saúde. In: MARQUES DE MELO, José (Org.). O campo da comunicação no Brasil. Petrópolis: Vozes, 2008.

PINHEIRO, Cynthia et al. Radiojornal Saúde e Você. In: INTERCOM NORTE, X, Boa Vista (RR), 01 a 03 jun. 2011. Anais... São Paulo: Intercom, 2011. Disponível em: <http://intercom.org.br/papers/regionais/norte2011/expocom/ EX26-0083-1. pdf>. Acesso em: 15 fev. 2012. 
RAMOS, Murilo C. Comunicação, direitos sociais e políticas publicas. In: Comunicação, direitos sociais e políticas públicas. In: MARQUES DE MELO, José; SATHLER, Luciano (Org.). Direitos à comunicação na sociedade da informação. São Bernardo do Campo (SP): Umesp, 2005. Disponível em: <http://www.lucianosathler.pro.br/wordpress/wp-content/uploads/2008/02/245_253_direitos_a_comunicacao_politicas_publicas_murilo_ramos.pdf>.Acesso em: 27 jan. 2012.

SILVA, Gislene et al. (Org.). Jornalismo contemporâneo: figurações, impasses e perspectivas. Salvador: Edufba / Compós, 2011.

TEIXEIRA, José A. Carvalho. Comunicação em saúde: relação técnicos de saúde-utentes. Aná - Análise Psicológica, v. 22, n. 3, p. 615-620, set. 2004. Disponível em http://www.scielo.oces.mctes.pt/pdf/aps/ v22n3/v22n3a21.pdf Acesso em 16 jan. 2012.

TRENCH, Belkis; ANTENOR, Samuel. Editorial. BIS - Boletim do Instituto de Saúde, São Paulo, v. 12, n. 1, abr. 2010. Disponível em: <http:// periodicos.ses.sp.bvs.br/scielo.php?script=sci_arttext\&pid=\$1518-18122010000100001\&lng=en\&nrm=iso>. Acesso em: 10 jan. 2012.

Recebido em: 20.03.2012 / Aceito em: 04.06.2012 